\title{
Modification of Leather Surface Using Low- Pressure Plasma and Antimicrobial Reagent
}

\author{
Sanja Ercegović Ražić ${ }^{1, *}$, Jadranka Akalović ${ }^{1}$, Tomislav Ivanković ${ }^{2}$ Anja Ludaš ${ }^{1}$ and \\ Katarina Ištef $^{1}$ \\ ${ }^{1}$ University of Zagreb, Faculty of Textile Technology, Department of Materials, Fibers and Textile \\ Testing, Prilaz baruna Filipovića 28a, 10000 Zagreb, Croatia \\ ${ }^{2}$ University of Zagreb, Faculty of Science, Department of Biology, Rooseveltov trg 610000 Zagreb, \\ Croatia
}

\begin{abstract}
Investigations, which are related to plasma efficiency on the treated leather surface, are significant in the development of ecologically and economically friendly processes in obtaining material of desired functional properties. Through the pretreatments using plasma different chemical-physical reactions in the surface layer of treated leather are occurred resulting in improved reactivity. In this paper, modification and functionalization of bovine leather using 1,2,3,4-butantetracarboxylic acid and chitosan were explored. Pretreatments of leather samples were realised using argon and oxygen plasma to assess various influence of chemically reactive oxygen and inert argon gas. Two different bovine leathers chrome tanned leather and leather tanned with synthetic tanning agent $(\mathrm{Cr}-$ free) were chosen for treatments. Analyse of the surface morphology was conducted with SEM microscopy, while the chemical changes using ATRFTIR spectroscopy. Antimicrobial effectiveness of treated leather was tested with qualitative Agar diffusion plate test against two bacterial Staphylococcus aureus and Klebsiella pneumoniae. Obtained results indicated how applied oxygen and argon plasma pretreatments in optimized process conditions contribute to the improvement of tested functional properties. Achieved surface changes positively affected on leather surface reactivity and antimicrobial effectiveness, particularly $\mathrm{Cr}$ free leather.
\end{abstract}

\section{Introduction}

The leather industry is one of the huge polluters of the environment, because of that it is important to develop environmentally friendly procedures. A major problem is the proven treatments, which are mainly carried out by conventional procedures, and are most often very harmful to the environment. The use of plasma as a medium for property modification is an acceptable technique because its application could completely replace, or at least shorten the processing time and use of large amounts of water and chemicals in order to achieve the desired properties of leather product. The main advantages of cold plasmas are

* Corresponding author: sanja.ercegovic@ttf.unizg.hr 
applicability to all kinds of materials without negative effects on the basic properties of the material, without high consumption of chemicals, water and energy. Plasma treatment impact to the modification surface layer of the treated leather, resulting in the surface cleaning and its activation thus achieving better hydrophilicity (absorptivity) for some new chemical reactions. At low-pressure plasma, it is possible to apply chemical agents in monomeric form, which have the ability to polymerize with the substrate, whereby cleavage or crosslinking of the agents on the surface can take place, in order to achieve and /or improve the functional properties [1].

Microorganisms have a significant, often negative impact on human health in the form of the development of unpleasant odours, stains and fungal diseases, so today there are a very large number of different antimicrobial agents. Recently, it has become important as an antimicrobial agent gaining chitosan due to its excellent biocompatibility and biodegradability, excellent antimicrobial properties and great availability in nature. Therefore, it was used in this work as an antimicrobial agent with environmentally friendly technology of cold low-pressure plasma on cowhide, which is coated with various agents in the production phase. The prepared chitosan solution was applied to treated bovine leather by the method of horizontal spraying of the agent with the aim of achieving antimicrobial efficacy against targeted gram-positive Staphylococcus aureus and gram-negative Klebsiella pneumonia bacterial species [2, 3].

Leather making is one of the oldest human crafts present in history for more than 3000 years. Humans used the leather of slaughtered animals to protect their bodies from the weather and injuries, and gradually for other purposes, such as making boats, tents, and other products [4]. The leather is one of the fundamental importance to the animal because it has various physiological functions, the most important of which are the regulation of body temperature, storage of nutrients, protection and excretion of waste products [5]. The waterproof layer of the leather acts as a barrier against various infections, preventing the absorption of water from the outside and the loss of water from the inside. Animal leather consists of the three main layers: epidermis, dermis and hypodermis. Dermis contains intertwined collagen fibers, which represent the most important layer of raw leather for obtaining the finished product, while the remaining two layers (epidermis and hypodermis) are removed during the processes (Fig. 1.) $[5,6]$.

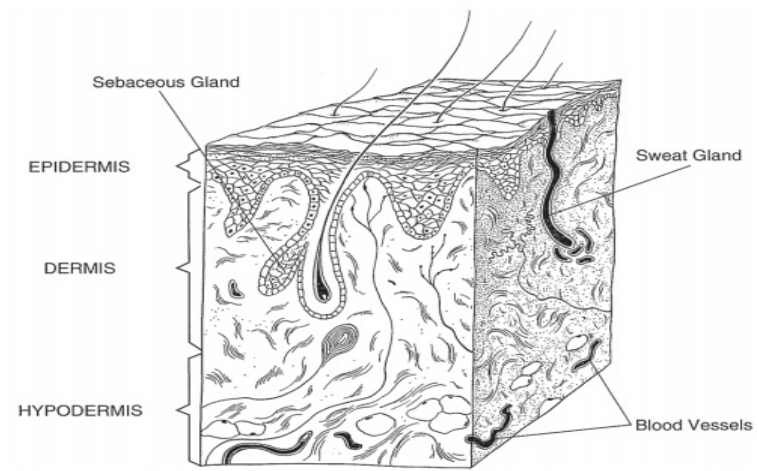

Fig. 1. Cross section of animal leather [6]

Finished leather is a product that is created by processing of raw animal leather in the tanning process by various means, of which the most widely used are chrome tanning, during which it becomes more durable and applicable. Tanning agents are agents that 
chemically react with the collagen molecule that builds the leather structure, stabilizing the triple helical structure of the collagen core, thus achieving leather resistance to chemical, thermal, and microbiological degradation. It has a complex morphological structure and its processing is extremely complex and requires the implementation of many process steps, of which the emphasis is on tanning in which it acquires its characteristic and unique properties and can be used for different purposes [5-7].

The emphasis of this paper is an introducing of environmentally friendly processes of the surface modification of semi-processed bovine tanned leathers, by application of plasma and ecological bio-agent chitosan, in order to achieve satisfactory functional (antimicrobial) properties of treated samples. The main goal is to achieve the desired properties by using of environmentally friendly processes with the lowest possible consumption of energy, water and chemicals to maximally preserve natural resources, with the purpose of protecting the environment and human health.

\section{Materials and Methods}

\subsection{Material}

The tests were carried out on two different semi-processed bovine leathers - chrome tanned leather and leather tanned with synthetic tanning agent ( $\mathrm{Cr}$-free). The basic characteristics of the tested leather samples are shown in Table 1.

Table 1. Basic characteristics of the examined leather samples

\begin{tabular}{|c|c|c|c|}
\hline $\begin{array}{c}\text { Sample name and } \\
\text { label }\end{array}$ & $\begin{array}{c}\text { Thickness } \\
{[\mathbf{m m}]}\end{array}$ & Sample description & $\begin{array}{c}\text { Sample face } \\
\text { appearance }\end{array}$ \\
\hline $\begin{array}{c}\text { Chrome tanned } \\
\text { leather (sample label: } \\
\text { Cr-leather) }\end{array}$ & $1,1-1,3 \mathrm{~mm}$ & $\begin{array}{c}\text { semi-processed bovine } \\
\text { chrome tanned and } \\
\text { hydrophobic leather full of } \\
\text { natural face }\end{array}$ & \\
\hline $\begin{array}{c}\text { Leather tanned with } \\
\text { synthetic tanning } \\
\text { agent (sample label: } \\
\text { Cr-free leather) }\end{array}$ & $1,1-1,3 \mathrm{~mm}$ & $\begin{array}{c}\text { semi-processed cowhide } \\
\text { with a full natural face, } \\
\text { synthetically tanned; } \\
\text { not hydrophobic }\end{array}$ & \\
\hline
\end{tabular}

\subsection{Methods of treatments}

\subsubsection{Plasma pretreatment}

Plasma pretreatments were realized using oxygen and argon gases (high purity $99.998 \%$, by Messer) at a defined parameters of pressure and gas flow rate, at constant frequency of 40 $\mathrm{kHz}$ in a low-pressure plasma system (NANO LF, Diener electronic), Table 2. For determination of the effect of exposure time and power on leather surface properties, time of 10,20 or 40 minutes under 500 and $800 \mathrm{~W}$ were explored. All samples were dried at 50 ${ }^{\circ} \mathrm{C}$ for 24 hours prior to plasma pretreatment to remove the moisture. Plasma pretreated samples were treated with BTCA immediately upon completion of pretreatment. 
Table 2. Plasma pretreatment conditions

\begin{tabular}{|c|c|c|c|c|c|c|}
\hline $\begin{array}{c}\text { Sample } \\
\text { label }\end{array}$ & Gas & $\begin{array}{r}\text { Time, } \\
\mathrm{t} \text { [min] } \\
\end{array}$ & $\begin{array}{c}\text { Power, } \\
\text { P [W] }\end{array}$ & $\begin{array}{c}\text { Pressure, } \\
\text { p [Pa] }\end{array}$ & $\begin{array}{c}\text { Gas flow, } \\
\text { q }\left[\mathrm{cm}^{3} / \mathrm{min}\right]\end{array}$ & $\begin{array}{c}\text { lxd } \\
{[\mathbf{m m}]}\end{array}$ \\
\hline Cr-leather & oxygen & 20 & 500 & \multirow{6}{*}{32} & \multirow{6}{*}{220} & \multirow{6}{*}{$100 \times 100$} \\
\hline Cr-leather & oxygen & 20 & 800 & & & \\
\hline Cr-free leather & oxygen & 10 & 500 & & & \\
\hline Cr-free leather & oxygen & 10 & 800 & & & \\
\hline Cr-leather & argon & 40 & 800 & & & \\
\hline Cr-free leather & argon & 40 & 500 & & & \\
\hline
\end{tabular}

* Frequency of low-pressure plasma system is constant - $40 \mathrm{kHz}$.

\subsubsection{Treatment with BTCA}

Polymerization process directly in the plasma chamber, was used with BTCA as reagent (in monomer bottle) at a pressure of $50 \mathrm{~Pa}$, gas flow of $200 \mathrm{~cm}^{3} / \mathrm{min}$ and frequency of $40 \mathrm{kHz}$ in a plasma system. The effects of exposure time for 30 minutes under the power of $100 \mathrm{~W}$, on the polymerization rate were explored.

\subsubsection{Spraying method with Chitosan}

Second part of the experiment was realised using $1 \%$ chitosan $\left(\mathrm{C}_{56} \mathrm{H}_{103} \mathrm{~N}_{9} \mathrm{O}_{39}\right.$, medium molecular weight $100-300 \mathrm{kDa}$ ) solution on the plasma pretreated and BTCA treated leather samples by spraying method for achievement of antibacterial effectiveness. For enhanced deposition process of antibacterial agent, leather was sprayed with chitosan solution for 5 second and dried at $65^{\circ} \mathrm{C}$ for 15 minutes.

\subsection{Methods of Analysis}

\subsubsection{Surface morphological analysis}

Surface changes of morphology were analysed using scanning electron microscope (SEM) (type JEOL LV-6060) at 100x and 2000x magnifications. The scanning electron microscope works by using a focused electron beam to scan the surface of the test sample to give information about the characteristics of the sample surface. When the electron beam is focused on the sample, various interactions occur, such as the emission of secondary and return primary electrons. A beam of electrons erupts electrons that are part of the cause atoms. The electron energies from the sample are collected and measured by detectors, and a pseudotrodimensional image is created with the help of a microprocessor. In order to obtain conductivity, samples were coated with gold for 20 minutes, using a sputter coater prior to analysis [8].

\subsubsection{Chemical changes}

The chemical changes were analysed using ATR-FTIR spectroscopy (Attenuated Total Reflectance-Fourier Transform Infrared Spectroscopy). Infrared spectroscopy is based on the interaction between IR radiation and molecules, i.e. groups of two or more atoms connected by a chemical bond. The material absorbs infrared radiation, and the amount of absorbed radiation is measured as a function of energy that can be expressed in the form of frequency. IR radiation is absorbed only if its frequency coincides with the vibration frequency of the molecular bond. The absorbed radiation is reflected as a "jump" on the spectrogram. The analysis of the samples on the device was performed by the method of 
Attenuated Total Reflectance (ATR), in which the IR light was completely reflected when it reached the surface of the ATR crystal. When the sample is placed on the crystal surface, the IR beam penetrates the sample to a certain depth depending on the angle of incidence of the beam and the refractive index of the ATR crystal, as well as the type of sample [9].

\subsubsection{Antimicrobial efficacy}

Antimicrobial effectiveness of treated leather samples was conducted using qualitative Agar diffusion plate test against two bacterial species Staphylococcus aureus (ATCC 25 923) and Klebsiella pneumonia (ATCC 11 296). The test procedure for antimicrobial efficacy of treated samples was performed in accordance with the standard HRN EN ISO 20645:2008, as well as the final assessment of antibacterial activity included the inhibition zone and the growth of the bacteria under the specimen.

\section{Results and discussion}

\subsection{SEM analysis of leather surface}

Based on the obtained images taken by scanning electron microscopy, the surface morphology characteristic of tested samples with observable follicles visible at a magnification of 100x is observed (Fig.2 a-d). By comparing images of the leather surface before and after pretreatment with $\mathrm{O}_{2}$ plasma at a magnification of $2000 \mathrm{x}$, a certain cleaning of the surface with residual surface "damage" of the face is observed (Fig. 2. b1). After treatment with polycarboxylic acid (BTCA) and chitosan on the pretreated surface with oxygen plasma (Fig. 2. c1 and d1), a slight smoothing and covering of the damaged parts with the applied agent was observed, which may indicate crosslinking of polycarboxylic acid on the sample surface and bonding.

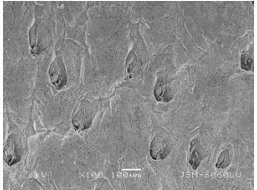

a) untreated

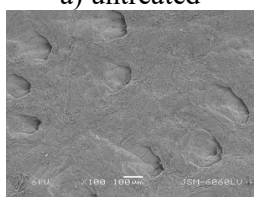

b) $\mathrm{O}_{2}$ plasma pretreated

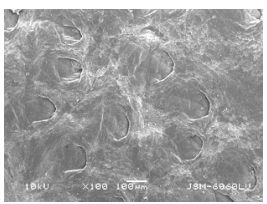

c) $\mathrm{O}_{2}$ /BTCA treatment

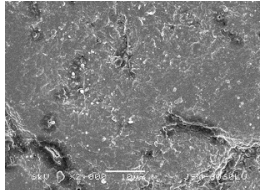

a1) untreated

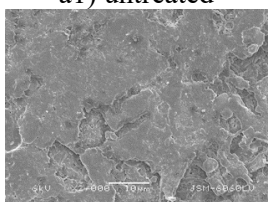

b1) $\mathrm{O}_{2}$ plasma pretreated

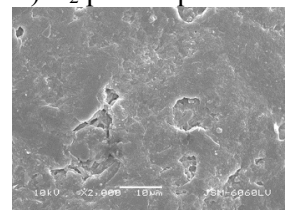

c1) $\mathrm{O}_{2}$ /BTCA treatment 


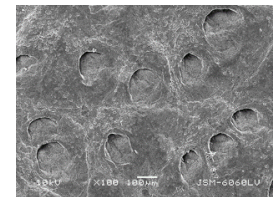

d) $\mathrm{O}_{2} / \mathrm{BTCA} / \mathrm{CH}$ treatment

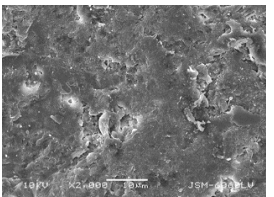

d1) $\mathrm{O}_{2} / \mathrm{BTCA} / \mathrm{CH}$ treatment

Fig. 2. SEM images of chrome tanned leather with 100x (left) and 2000x (right) magnifications: a, a1) untreated; b, b1) $\mathrm{O}_{2}$ plasma pretreated; c, c1) $\mathrm{O}_{2} / \mathrm{BTCA}$ treatment and d, d1) $\mathrm{O}_{2} / \mathrm{BTCA} / \mathrm{CH}$ treatment

By analysing microscopic images of chromium tanned samples, after pretreatment with argon plasma, granular segments are visible on the surface of tested sample, and the surface is rougher with more pronounced and open follicles. Treatment with chitosan after pretreatment with Ar plasma makes the surface smooth and the follicles are closed (Fig. 3. $\mathrm{b}$ and $\mathrm{b} 1)$. After treatments with BTCA and chitosan, an increased amount of agent is observed on the leather surface (Fig. 3. c1).

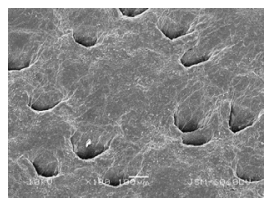

a) Ar plasma pretreated

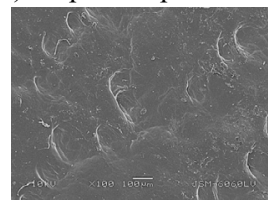

b) $\mathrm{Ar} / \mathrm{CH}$ treatment

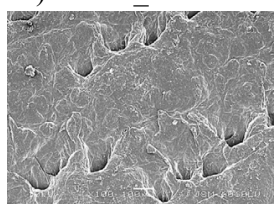

c) $\mathrm{Ar} / \mathrm{BTCA} / \mathrm{CH}$ treatment

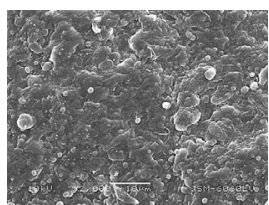

a1) Ar plasma pretreated

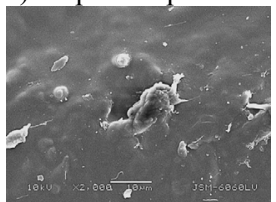

b1) $\mathrm{Ar} / \mathrm{CH}$ treatment

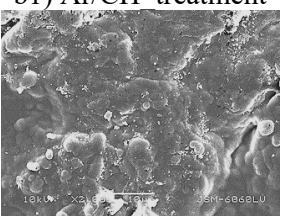

c1) $\mathrm{Ar} / \mathrm{BTCA} / \mathrm{CH}$ treatment

Fig. 3. SEM images of a chromium tanned leather sample with magnifications of $100 \mathrm{x}$ (left) and 2000x (right): a, a1) argon plasma; b, b1) $\mathrm{Ar} / \mathrm{CH}$ treatment and c, c1) $\mathrm{Ar} / \mathrm{BTCA} / \mathrm{CH}$ treatment

By analysing the surface of the tanned sample with synthetic tanning agent, after pretreatment with oxygen plasma (Fig. 4), the surface of the sample was cleaned of present impurities visible on the untreated sample (Fig. 4. a1), but also additionally "damaged" surface layer (face) below which collagen fibres (Fig. 4. b1). After the treatments with BTCA and chitosan, the surface is closed, smoothed and covered with the visible content of the applied agent (Fig. 4. c1, d1). 


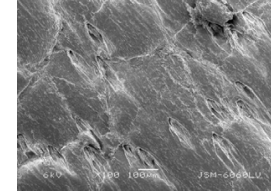

a) untreated

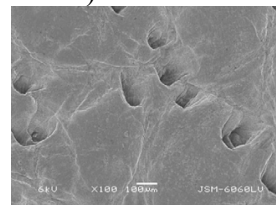

b) $\mathrm{O}_{2}$ plasma pretreated

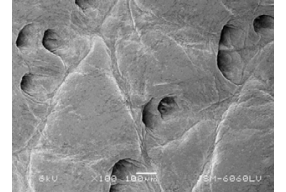

c) $\mathrm{O}_{2}$ /BTCA treatment

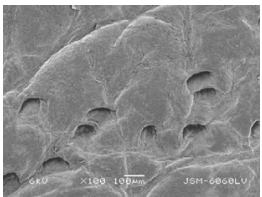

d) $\mathrm{O}_{2} / \mathrm{BTCA} / \mathrm{CH}$ treatment

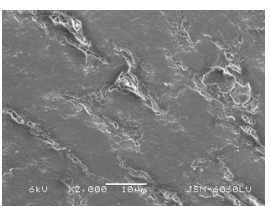

a1) untreated

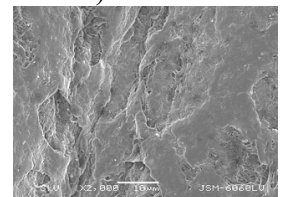

b1) $\mathrm{O}_{2}$ plasma pretreated
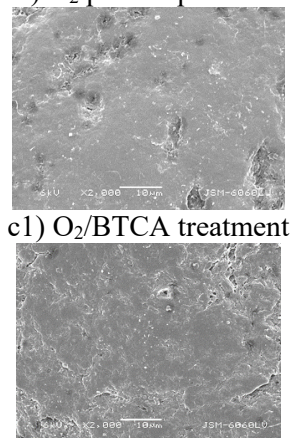

d1) $\mathrm{O}_{2} / \mathrm{BTCA} / \mathrm{CH}$ treatment

Fig. 4. SEM images of synthetic tanned leather with 100x (left) and 2000x (right) magnifications: a, a1) untreated; b, b1) $\mathrm{O}_{2}$ plasma pre-treated; c, c1) $\mathrm{O}_{2} / \mathrm{BTCA}$ treatment and d, d1) $\mathrm{O}_{2} / \mathrm{BTCA} / \mathrm{CH}$ treatment

By microscopic analysis of the surface of the tanned sample with synthetic tanning agent, after pretreatment with argon plasma, the surface of the sample is more open in the presence of surface craters formed due to the action of argon gas (Fig. 5. b1). After application of the crosslinking agent BTCA and antibacterial agent chitosan, the agents bound to the collagen fibres of the sample, which is visible at image with magnification of 2000x after treatment Ar/BTCA/CH (Fig. 5. c1).

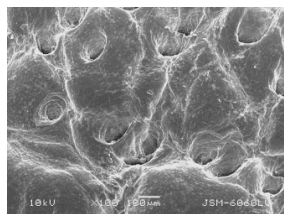

a) Ar plasma pretreated

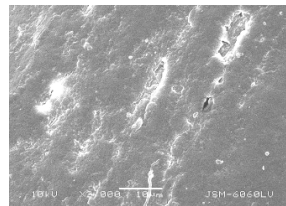

a1) Ar plasma pretreated 


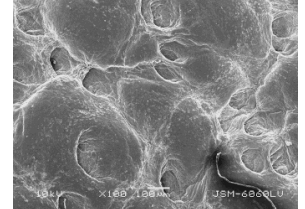

b) $\mathrm{Ar} / \mathrm{CH}$ treatment

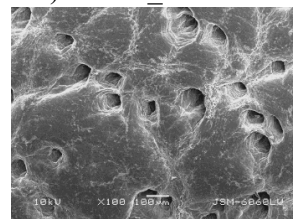

c) $\mathrm{Ar} / \mathrm{BTCA} / \mathrm{CH}$ treatment

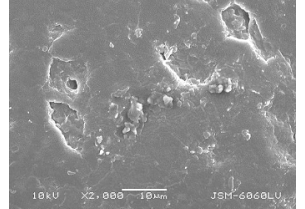

b1) $\mathrm{Ar} / \mathrm{CH}$ treatment

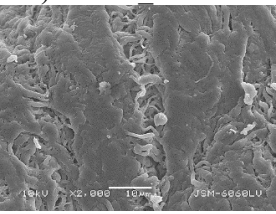

c1) $\mathrm{Ar} / \mathrm{BTCA} / \mathrm{CH}$ treatment

Fig. 5. SEM images of synthetic tanned leather with 100x (left) and 2000x (right) magnifications: a, a1) argon plasma; b, b1) $\mathrm{Ar} / \mathrm{CH}$ treatment and c, c1) $\mathrm{Ar} / \mathrm{BTCA} / \mathrm{CH}$ treatment

\subsection{Results of FTIR-ATR functional group analysis}

The chemical structure of the surface of untreated and treated samples was analysed, i.e. the presence of characteristic wave bands (peaks) corresponding to functional groups. For better comparison, each graph shows the spectra of untreated samples and the spectra of samples after plasma pretreatments/BTCA and chitosan treatments in the spectral range of the mean IR spectrum from $4000 \mathrm{~cm}^{-1}$ to $600 \mathrm{~cm}^{-1}$.

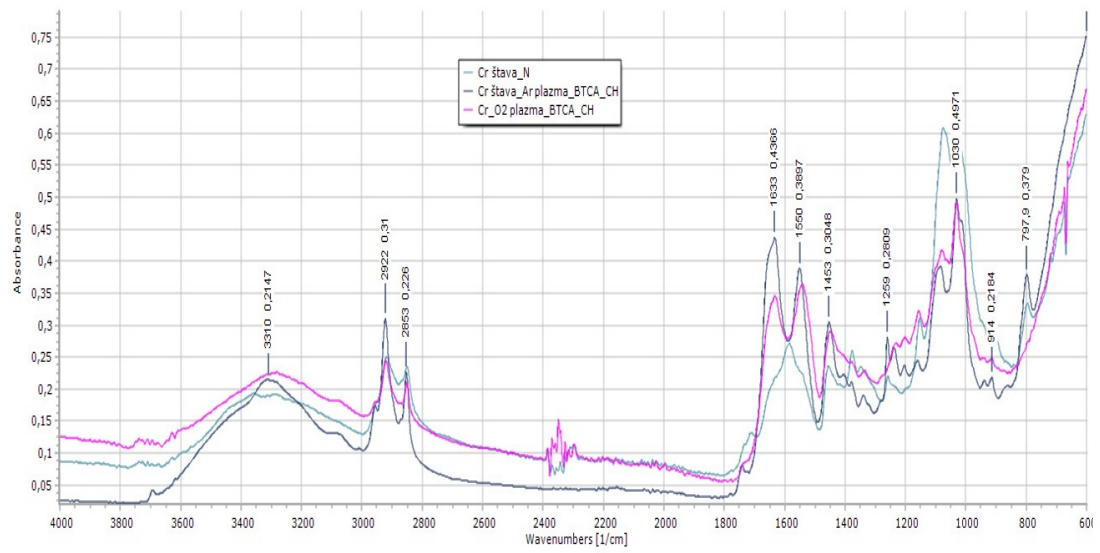

Fig. 6. Absorption FTIR spectra of chromium tanned leather sample before and after treatment with $\mathrm{O}_{2}$ or $\mathrm{Ar} / \mathrm{BTCA} / \mathrm{CH}$

By comparing the spectra shown in Fig. 6., the intensity of the absorption bands of the treated samples is observed in relation to the untreated ones at $2922 \mathrm{~cm}^{-1}, 1633 \mathrm{~cm}^{-1}, 1550$ $\mathrm{cm}^{-1}, 1453 \mathrm{~cm}^{-1}$ and $797 \mathrm{~cm}^{-1}$, which indicate that intense vibrations occur on the $-\mathrm{NH}$, $\mathrm{NH}_{2},-\mathrm{CH},-\mathrm{CH}_{2}$ groups and $-\mathrm{C}=\mathrm{O}$ groups, which show that polycarboxylic acid (BTCA) and chitosan are very likely to bind to the substrate surface. The presence of absorption 
peaks of the treated samples at $1633 \mathrm{~cm}^{-1}$ and $1550 \mathrm{~cm}^{-1}$, which are not present in the untreated sample, and which correspond to the $-\mathrm{C}=\mathrm{O}$ and $-\mathrm{NH}_{2}$ groups is observed. That may indicate the binding of chitosan to the substrate surface. There is also a decrease in the intensity of the absorption peak at $1030 \mathrm{~cm}^{-1}$, which indicates reduced tensile vibrations in the $-\mathrm{C}=\mathrm{O}$ group $[10-13]$.

The FTIR-ATR spectrum of the tanned sample with synthetic tanning agent (Fig. 7.) has marked absorption bands in the spectral range at characteristic wavenumbers where at 3311 $\mathrm{cm}^{-1}$ and $3083 \mathrm{~cm}^{-1}$ they indicate tensile vibrations $-\mathrm{NH}$ group of amino acids, at $2921 \mathrm{~cm}^{-1}$ $-\mathrm{CH}$ asymmetric deformation vibrations, at $1630 \mathrm{~cm}^{-1}$ tensile vibrations $-\mathrm{C}=\mathrm{O}$ group. The higher intensity of the absorption bands of the processed samples was expressed at wavenumbers at $2921 \mathrm{~cm}^{-1}$ for $-\mathrm{CH}$ groups, at $2852 \mathrm{~cm}^{-1}-\mathrm{CH}_{2}$ groups, at $1630 \mathrm{~cm}^{-1}$ for $\mathrm{C}=\mathrm{O}$ groups, at $1551 \mathrm{~cm}^{-1}$ for $-\mathrm{NH}_{2}$ deformation vibrations, at $1315 \mathrm{~cm}^{-1}$ for $-\mathrm{COC}-$ tensile vibrations, at $1236 \mathrm{~cm}^{-1}$ for $-\mathrm{C}=\mathrm{O}$ groups and at $780 \mathrm{~cm}^{-1}$ for aromatic $-\mathrm{CH}$ groups. The intensity of the absorption bands of the sample treated with argon plasma, with BTCA and chitosan is higher at $1630 \mathrm{~cm}^{-1}, 1551 \mathrm{~cm}^{-1}$ and $780 \mathrm{~cm}^{-1}$, which may indicate better binding of chitosan and BTCA to the substrate after pretreatment with argon plasma. The intensity of the absorption bands at the wavenumbers at $1158 \mathrm{~cm}^{-1}, 1103 \mathrm{~cm}^{-1}$ and $1032 \mathrm{~cm}^{-1}$, which indicate reduced vibrations at $-\mathrm{C}=\mathrm{O}$, and $-\mathrm{CH}$ groups, was reduced [10-13].

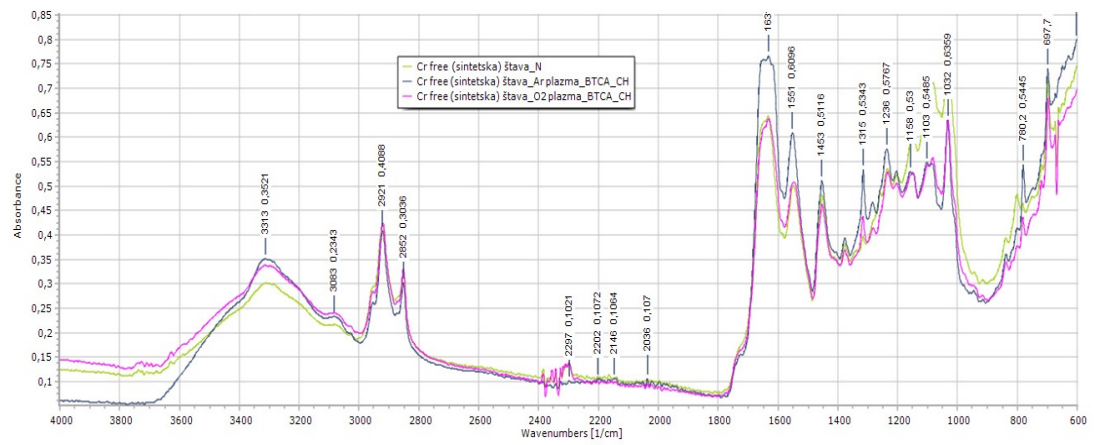

Fig. 7. Absorption FTIR spectra of a synthetic tanned leather sample before and after treatment with $\mathrm{O}_{2}$ or $\mathrm{Ar} / \mathrm{BTCA} / \mathrm{CH}$

\subsection{Antibacterial activity against selected bacteria}

The results of antimicrobial efficacy of untreated and treated leather samples against specified bacteria Staphylococcus aureus and Klebsiella pneumoniae are shown in Table 3.

Table 3. Antibacterial activity of untreated and treated leather samples.

\begin{tabular}{|c|c|c|c|}
\hline \multirow{2}{*}{ Sample label } & Treatment & Growth under the specimen & Assessment* \\
\hline \multicolumn{3}{|c|}{ Staphylococcus aureus } \\
\hline \multirow{3}{*}{ Cr-leather } & Untreated & slight & limit of efficacy \\
\cline { 2 - 4 } & $\mathrm{O}_{2} / \mathrm{BTCA} / \mathrm{CH}$ & none & good effect \\
\cline { 2 - 4 } & $\mathrm{Ar} / \mathrm{BTCA} / \mathrm{CH}$ & slight & limit of efficacy \\
\hline \multirow{2}{*}{ Cr-free leather } & Untreated & none & good effect \\
\cline { 2 - 4 } & $\mathrm{O}_{2} / \mathrm{BTCA} / \mathrm{CH}$ & none &
\end{tabular}




\begin{tabular}{|c|c|c|c|}
\hline \multirow{2}{*}{} & $\mathrm{Ar} / \mathrm{BTCA} / \mathrm{CH}$ & none & good effect \\
\hline \multirow{3}{*}{ Cr-leather } & \multicolumn{2}{|c|}{ Klebsiella pneumoniae } \\
\cline { 2 - 4 } & Untreated & none & good effect \\
\cline { 2 - 4 } & $\mathrm{O}_{2} / \mathrm{BTCA} / \mathrm{CH}$ & none & good effect \\
\hline \multirow{3}{*}{ Cr-free leather } & $\mathrm{Ar} / \mathrm{BTCA} / \mathrm{CH}$ & medium & good effect \\
\cline { 2 - 4 } & Untreated & none & good effect \\
\cline { 2 - 4 } & $\mathrm{O}_{2} / \mathrm{BTCA} / \mathrm{CH}$ & none & good effect \\
\cline { 2 - 4 } & $\mathrm{Ar} / \mathrm{BTCA} / \mathrm{CH}$ & none & inent
\end{tabular}

*Assessment is based on HRN EN ISO 20645:2008 - "Determination of antibacterial activity - Agar diffusion plate test", where "good effect" is described as "no growth" under the specimen, "limit of efficacy" as "slight growth (only some restricted colonies or growth nearly totally suppressed)" under the specimen and "insufficient effect" is described as "growth" of bacterial colonies under the specimen.

Both of samples show a good effect against Staphylococcus aureus after treatments. Tanned sample with synthetic tanning agent show significant antimicrobial activity prior to plasma pretreatment and treatment with agents (BTCA and chitosan). In the untreated chromium tanned sample there is a slight growth and without present zone of inhibition, which indicates that the antimicrobial effect is limited. Although, after treatment with plasmas and agents, zone of inhibition is not evident, bacterial growth below the sample is not present indicating good antimicrobial effect. The chromium tanned sample after pretreatment with argon plasma and treatment with the agents resulting in growing of tested bacteria, and it's assumed that no chitosan particles have bound to its surface, or the sample is contaminated during the antibacterial testing.

The obtained results in which the antimicrobial effect of the treated leather samples against the negative bacteria Klebsiella pneumoniae were tested show a generally good antimicrobial effect, which is manifested without bacterial growth and without a visible zone of bacterial inhibition. Slightly poorer results are shown by the chromium tanned sample after pretreatment with argon plasma and treatment with agents whose presence reduced its antimicrobial efficacy.

\section{Conclusions}

Morphological changes of leather samples after pretreatment with oxygen and argon plasma were confirmed by analysis of SEM images where the 'cleaning' of the surfaces of the chromium tanned and $\mathrm{Cr}$-free tanned sample is visible. After treatment with polycarboxylic acid (BTCA) and chitosan on pretreated plasma surfaces, a slight smoothing and covering of the damaged parts with applied agents is visible, which may indicate on crosslinking of polycarboxylic acid on the sample surface and binding of chitosan particles.

By analyzing the chemical structure of the surfaces of untreated and treated samples using FTIR-ATR spectroscopy, absorption peaks at $1630 \mathrm{~cm}^{-1}$ and $1550 \mathrm{~cm}^{-1}$ corresponding to the $-\mathrm{C}=\mathrm{O}$ and $-\mathrm{NH}_{2}$ groups are observed. An increase in the intensity of the absorption peaks may indicate the binding of chitosan to the surface of the substrate.

The applied treatments achieved satisfactory antimicrobial efficacy of both tested leather samples, especially the $\mathrm{Cr}$-free tanned sample after pretreatment with oxygen and argon plasma and treatment with chitosan as antibacterial agent.

This work has been fully supported by the Croatian Science Foundation under the project (IP-201606-5278). Work (SEM analysis) was also financed by Slovenian Finance Agency, Slovenia (Infrastructure Centre RIC UL-NTF). 


\section{References}

1. Kaygusuz, M. et al:: Modification of Leather Surface with Atmospheric Pressure Plasma and Nano-Finishing, Polymer-Plastics Technology and Engineering, 57 (2018) 4, 260-268, ISSN 2574-0881.

2. Li, Q., E. T. Dunn, E. W. Grandmaison, M. F. A. Goosen: Applications and properties of chitosan, Journal of Bioactive and Compatible Polymers, 7 (1992) 370-397

3. Rinaudo, M.: Chitin and chitosan: Properties and applications, Progress in Polymer Science, 31 (2006) 603-632

4. Reed, R: Science for students of leather technology, Pergamon Press Ltd., Headington Hill Hall, Oxford (1966) 5, 134-138

5. Nazer, D.W., Al-Sa'ed, R.M., Siebel, M.A.: Reducing the environmental impact of the unhairing-limingprocess in the leather tanning industry, Journal of Cleaner Production, 14 (2006) $65-74$

6. Sizeland, K.: Nanostructure and physical properties of collagen biomaterials, Doctoral thesys, Massey University, Manawatu, New Zealand, 2015

7. Štěpánová, V. et al.: Surface modification of natural leather using diffuse ambient air plasma, International Journal od Adhesion and Adhesives, 77 (2017) 198-203, ISSN 01437496.

8. Mršić, G. \& Žugaj, S.: Analiza GSR čestica upotrebom elektronskog mikroskopa (SEM/EDX), Policija i sigurnost, 16 (2008) 3-4, 179-200.

9. Zofka, A., Maliszewska, D., Maliszewski, M., Boratynski, J.: Application of FTIR method to examine the polymer content in the modified bitumen and to assess susceptibility of bitumen to ageing, Roads and Bridges - Drogi i Mosty, 14 (2015) $163-174$

10. Hedberg, Y.S., Lidén, C., Odnevall Wallinder, I.: Correlation between bulk- and surface chemistry of Cr-tanned leatherand the release of $\mathrm{Cr}(\mathrm{III})$ and $\mathrm{Cr}(\mathrm{VI})$, Journal of Hazardous Materials 280 (2014) 654-661

11. Falcão, L., Araújo M.E.M.: Application of ATR-FTIR spectroscopy to the analysis of tannins inhistoric leathers: The case study of the upholstery from the 19thcentury Portuguese Royal Train, Vibrational Spectroscopy74 (2014) 98-103

12. Nashy E.H.A., Osman, O., Mahmoud, A.A., Ibrahim, M: Molecular spectroscopic study for suggested mechanism of chrome tanned leather, Spectrochimica Acta Part A88 (2012) 171- 176

13. Tite, T., Miu, L., Giurginca, M., Meghea, A.: Evaluation of degradation of parchment leather by FT-IR and UV-VIS-NIR spectroscopy, Revista de Chimie57 (5) (2006) 466-471. 